\title{
Short-term outcomes of mirogabalin in patients with peripheral neuropathic pain: a retrospective study
}

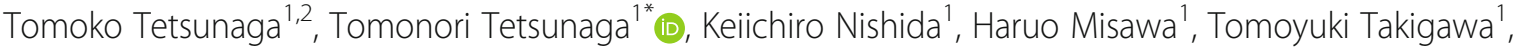 \\ Kentaro Yamane ${ }^{1}$, Hironori Tsuji ${ }^{1}$ Yoshitaka Takei ${ }^{2}$ and Toshifumi Ozaki ${ }^{1}$
}

\begin{abstract}
Background: Mirogabalin, which is approved for the treatment of peripheral neuropathic pain in Japan, is a ligand for the a $2 \delta$ subunit of voltage-gated calcium channels. Both pregabalin and mirogabalin act as nonselective ligands at the $a 2 \delta-1$ and a2 $\delta-2$ subunits. Mirogabalin has a unique binding profile and long duration of action. Pregabalin has been reported to produce intolerable adverse effects in some patients. This study investigated outcomes associated with mirogabalin administration in patients with peripheral neuropathic pain who ceased treatment with pregabalin.
\end{abstract}

Methods: We retrospectively assessed peripheral neuropathic pain using the neuropathic pain screening questionnaire (NeP score) in 187 patients (58 men, 129 women) who were treated with mirogabalin. All patients had switched from pregabalin to mirogabalin due to lack of efficacy or adverse events. Differences in the treatment course (i.e., numeric rating scale (NRS) scores) were compared using one-way analysis of variance with Bonferroni post hoc tests.

Results: The mean age of the patients was 72.3 years (range, 30-94 years), and the mean duration of disease was 37 months (range, 3-252 months). After treatment with mirogabalin for 1 week, NRS scores significantly decreased compared with baseline and continued to decrease over time. After 8 weeks, NRS scores improved by $\geq 30 \%$ from baseline in 113 patients (69.3\%). Twenty-four patients (12.8\%) stopped mirogabalin treatment due to adverse events. Somnolence (26.7\%), dizziness (12.3\%), edema (5.9\%), and weight gain (0.5\%) were noted as adverse events of mirogabalin.

Conclusions: The results of this investigation indicate that mirogabalin is safe and effective for reducing peripheral neuropathic pain.

Keywords: Peripheral neuropathic pain, Mirogabalin, Pregabalin, Adverse event

\section{Background}

The International Association for the Study of Pain defined neuropathic pain as "pain caused by a lesion or disease of the somatosensory nervous system" [1]. Neuropathic pain results in multiple symptoms, including spontaneous neurological pain, allodynia, hyperalgesia,

\footnotetext{
* Correspondence: tomonori_t31@yahoo.co.jp

'Department of Orthopedic Surgery, Okayama University Hospital, Okayama, Japan, 2-5-1 Shikata-cho, Kitaku, Okayama City, Okayama 700-8558, Japan Full list of author information is available at the end of the article
}

and numbness, and results in decreases in quality of life (QOL) [2]. In addition, neuropathic pain may become intractable [2]. Chronic low back pain is known to be due to neuropathic as well as nociceptive pain mechanisms [3, 4]. Patients with neuropathic pain show higher ratings for pain intensity with more comorbidities, such as depression panic/anxiety disorder, and sleep disorders than those with nociceptive pain [4]. It is thus important to determine which factors contribute to neuropathic pain at an early stage and start appropriate drug therapy [5]. 
Unfortunately, current treatment methods are not always satisfactory [5].

Pregabalin is a ligand for the $\alpha 2 \delta$ subunit of voltagesensitive calcium channels and is recommended as the first-line drug for neuropathic pain in guidelines around the world [6]. It decreases the release of neurotransmitters such as glutamate, noradrenalin, and substance P, which leads to pain relief [7]. Pregabalin has been used in patients with neuropathic pain and shown to be a cost-effective treatment $[8,9]$ that has a positive impact on QOL $[10,11]$. It is generally well tolerated [12], and most adverse events are mild to moderate [13]. Pregabalin binds to the $\alpha 2 \delta-1$ and $\alpha 2 \delta$ 2 subunits of presynaptic, voltage-dependent calcium channels, which are widely distributed throughout the central and peripheral nervous systems [14]. The most frequently reported side effects of pregabalin include dizziness and somnolence, which are related to the central nervous system (CNS) [14]. Therefore, the clinical utility of pregabalin may be limited by $\mathrm{CNS}$ adverse events [14].

Mirogabalin, which is a potent and specific ligand for the $\alpha 2 \delta$ subunit of voltage-gated calcium channels, is an orally administered gabapentinoid developed for the treatment of peripheral neuropathic pain in Japan [15, 16]. This agent has the distinguishing feature of persistently binding to the $\alpha 2 \delta-1$ subunit, which plays an important role in neuropathic pain [16]. This new drug is reportedly well tolerated and well absorbed following oral administration and was first approved for peripheral neuropathic pain in 2019.

Although there are many positive reports associated with pregabalin, some patients have moderate adverse events [14]. Tetsunaga et al. reported that somnolence (46.9\%), dizziness (18.8\%), weight gain $(9.4 \%)$, and rash $(4.7 \%)$ were noted as side effects of pregabalin [17]. No reports regarding the treatment outcomes with mirogabalin in patients who experienced moderate adverse events with pregabalin treatment have been published. In the present study, we examined the outcomes with mirogabalin as a rescue drug in patients with peripheral neuropathic pain who developed moderate side effects from pregabalin treatment.

\section{Methods}

\section{Participants}

This retrospective study included outpatients with peripheral neuropathic pain who consulted our hospital between April 2019 and October 2019. Data were collected from medical records. The diagnosis of peripheral neuropathic pain was based on a history of neuropathic pain and confirmatory findings on examination. The inclusion criteria were a diagnosis of peripheral neuropathic pain based on the flow chart of the grading system for neuropathic pain [18], lack of efficacy with pregabalin (Lyrica ${ }^{\circ}$, Pfizer Inc., Tokyo, Japan) or adverse events with pregabalin treatment, and the willingness to answer a questionnaire. The exclusion criteria included dementia, delirium, or other conditions that made it difficult to complete a self-reported written questionnaire. Patients with severe chronic diseases that interfered with treatment (e.g., cardiovascular disease, renal failure, or other disqualifying conditions) were also excluded. At baseline, the patients completed a self-reported questionnaire and provided demographic and clinical information. This study was approved by the Kurashiki Municipal Hospital ethics committee, and written informed consent was waived because of the retrospective design.

\section{Procedure}

\section{Treatment protocol}

At least 1 month after cessation from pregabalin, treatment with mirogabalin (Tarlige ${ }^{\bullet}$, Daiichi Sankyo, Inc., Tokyo, Japan) was prescribed. The patients received mirogabalin $10 \mathrm{mg} /$ day orally for the first week. In patients with decreased renal function, the dose of mirogabalin was decreased to $5 \mathrm{mg} /$ day. Depending on patient age and symptoms, the dose of mirogabalin was decreased or increased as required to between $2.5 \mathrm{mg}$ and $15 \mathrm{mg} /$ dose twice daily. All patients visited the hospital at 1, 2, 4 , and 8 weeks to ensure compliance with the study regimen. The patients with an adequate effect received the same dose of mirogabalin. In patients with an inadequate effect, the dose of mirogabalin was increased up to 30 $\mathrm{mg} /$ day. If adverse events were observed, the dose was decreased. In this study, no other conservative treatments (nonsteroidal anti-inflammatory drugs and rehabilitation) or surgeries were performed. During the study, only the dispensing pharmacist had knowledge of the patient codes. The manufacturer and provider of mirogabalin (Daiichi Sankyo, Inc., Tokyo, Japan) was not involved in the protocol development, data collection and management, statistical analysis, or manuscript preparation.

\section{Clinical assessment}

The neuropathic pain screening questionnaire $(\mathrm{NeP}$ score), developed by Ogawa et al., was used for the peripheral neuropathic pain survey (Table 1) [19-21]. The patients' answers to questions in seven domains were weighted and scored. The likelihood of neuropathic pain was determined based on the total score as follows: $\geq$ $5=$ highly likely to have neuropathic pain; $4=$ likely to have neuropathic pain; $3=$ possibility of neuropathic pain; $\leq 2=$ unlikely to have neuropathic pain. A score $\geq 4$ was judged as representing neuropathic pain [20]. The numeric rating scale (NRS) for pain self-assessment is a 
Table 1 Neuropathic pain screening questionnaire

\begin{tabular}{l}
\hline Question \\
\hline Q1
\end{tabular}

Each of the items is scored on a 5 -point scale $(0=$ never; 1 = slight; $2=$ moderate; 3 = strong; $4=$ very strong). The total score can range from 0 to 28 points, with higher scores indicating greater pain.

widely used, valid, and reliable tool to measure chronic pain intensity [22]. The scores ranged from 0 to 10 , with 0 representing no pain and 10 representing the worst pain imaginable. The NRS scores were obtained at baseline and at 1,2, 4, and 8 weeks of treatment. We also evaluated adverse events of mirogabalin.

\section{Primary endpoint}

The primary endpoint of this study was to analyze the treatment course of peripheral neuropathic pain using NRS scores in patients receiving mirogabalin.

\section{Secondary endpoint}

The secondary endpoint of the current study was to investigate the adverse events of mirogabalin.

\section{Statistical analysis}

Factors associated with the cessation of mirogabalin treatment due to adverse events were identified using univariate analyses between the patients who continued treatment and those who ceased from treatment. Differences in the treatment course (i.e., NRS scores) were compared using one-way analysis of variance with Bonferroni post hoc tests. Moderate improvements in pain are considered to be $30 \%$ h Bonferroni postnivariate $50 \%$ pain relief is considered a good outcome [23, 24]. Therefore, we divided patients into two groups based on pain relief levels of $<30 \%$ or $\geq 30 \%$ after 8 weeks of treatment with mirogabalin. Normally distributed variables were compared using Student's t-tests, and non-normally distributed variables were compared using Mann-Whitney $\mathrm{U}$ tests. ables were compared using d using Studentpain relief levels of <analyses between ain using NRS scores in pabetween groups. Differences in the magnitude of NRS improvement between the groups with initial doses of $5 \mathrm{mg} /$ day and $10 \mathrm{mg} /$ day were compared using Student's t-tests. Differences in the magnitude of NRS improvement in patients taking maximum doses were compared using one-way analysis of variance. Differences of $p<0.05$ were considered significant. Statistical analyses were conducted using SPSS software version 25.0 for Windows (IBM Corporation, Armonk, NY, USA).

\section{Results \\ Participants}

Of the 337 outpatients with peripheral neuropathic pain who had been treated with pregabalin, 187 (55.5\%) met the inclusion criteria and were included in this study (Table 2). Adverse events leading to the switch from pregabalin to mirogabalin included somnolence (97 patients, $28.8 \%$ ), dizziness (50 patients, $14.8 \%$ ), edema (7 patients, $2.1 \%$ ), weight gain (3 patients, $0.9 \%$ ), epigastralgia ( 2 patients, $0.6 \%$ ), and fatigue ( 2 patients, $0.6 \%$ ). Thirty-two patients switched to mirogabalin due to a lack of efficacy with pregabalin. This study included 58 men and 129 women with a mean age of 72.3 years (range, $30-94$ years) at the time of the baseline examination. The mean pain duration from onset until consultation was 37 months (range, 3-252 months). In this study, 134 patients had lumbar canal stenosis, 33 had cervical spondylotic myelopathy, 10 had lumbar disc herniation, 9 had carpal tunnel syndrome, and 1 had postoperative pain.

\section{Treatment with mirogabalin}

Fifty-nine patients received mirogabalin $10 \mathrm{mg} /$ day orally for the first week, and 128 patients received mirogabalin $5 \mathrm{mg} /$ day due to decreased renal function. Thirty-three patients were treated with $5 \mathrm{mg} /$ day for 8 weeks of treatment. Eighty-one patients increased the dose of mirogabalin to $10 \mathrm{mg} /$ day due to lack of efficacy. Thirty patients increased the dose of mirogabalin to $20 \mathrm{mg} /$ day. Nineteen patients increased the dose of mirogabalin to $30 \mathrm{mg} /$ day; none of these patients experienced severe adverse events with the increased dose. Twenty-four patients $(12.8 \%)$ withdrew from mirogabalin treatment

Table 2 Patient characteristics

\begin{tabular}{ll}
\hline Variables & $\boldsymbol{n}=187$ \\
\hline Age (years) & $72.3 \pm 12.4$ (30-94) \\
Sex, female/male & $129 / 58$ \\
Diagnosis & \\
LCS & 134 \\
CSM & 33 \\
LDH & 10 \\
CTS & 9 \\
Others & 1 \\
BMI (kg/m $\left.{ }^{2}\right)$ & $23.1 \pm 4.2(18-33)$ \\
DM & 17
\end{tabular}

Data are expressed as the mean \pm standard deviation (range) or $n(\%)$ LCS lumbar canal stenosis, CSM cervical spondylotic myelopathy, $L D H$ lumbar disc herniation, CTS carpal tunnel syndrome, BMI body mass index, DM diabetes mellitus 
because of adverse events. Adverse events associated with mirogabalin included somnolence (50 patients, 26.7\%), dizziness (23 patients, $12.3 \%$ ), edema (11 patients, 5.9\%), epigastric pain (2 patients, $1.1 \%$ ), weight gain ( 1 patient, $0.5 \%$ ), and fatigue (1 patient, $0.5 \%)$. We investigated factors associated with the cessation of mirogabalin treatment due to adverse events. There were no significant differences in age, sex, body mass index (BMI), or diagnosis between the patients who discontinued mirogabalin and those who continued its use (Table $3)$. The patients who discontinued mirogabalin had a significantly lower incidence of somnolence $(p=0.0017)$ and significantly higher incidences of dizziness $(p=$ $0.015)$ and edema $(p=0.012)$ with pregabalin than those who continued mirogabalin. The patients who discontinued mirogabalin also had significantly higher incidences of dizziness $(p=0.0069)$ and edema $(p=0.016)$ than the patients who continued mirogabalin, and these effects contributed to the cessation of treatment.

We also investigated changes in NRS scores over the course of treatment with mirogabalin for 8 weeks. After treatment with mirogabalin for 1 week, NRS scores significantly decreased compared with baseline $(p<0.0001$, Fig. 1) and subsequently continued to decrease over time. After 8 weeks, NRS scores had improved by $\geq 30 \%$ compared with baseline in 113 patients (69.3\%). We divided the patients into two groups to identify factors associated with improvements in NRS scores of $<30 \%$ and $\geq 30 \%$ after 8 weeks (Table 4 ). There were no significant differences in age, sex, diagnosis, or BMI between groups, but the NeP score was significantly higher in patients who experienced pain relief $<30 \%$ than in those

Table 3 Univariate analyses comparing factors associated with continuation or withdrawal of treatment with mirogabalin

\begin{tabular}{|c|c|c|c|}
\hline Variables & $\begin{array}{l}\text { Continued treatment } \\
(n=163)\end{array}$ & $\begin{array}{l}\text { Withdrew from treatment } \\
(n=24)\end{array}$ & $p$ value \\
\hline Age (years) & $71.8 \pm 12.7(30-94)$ & $75.0 \pm 9.9(49-91)$ & $0.23^{\mathrm{a}}$ \\
\hline Sex, female/male & $110 / 53$ & $19 / 5$ & $0.25^{b}$ \\
\hline Diagnosis & & & $0.98^{b}$ \\
\hline LCS & 118 & 17 & \\
\hline CSM & 28 & 5 & \\
\hline $\mathrm{LDH}$ & 9 & 1 & \\
\hline CTS & 8 & 1 & \\
\hline Others & 1 & 0 & \\
\hline BMI $\left(\mathrm{kg} / \mathrm{m}^{2}\right)$ & $22.9 \pm 4.1(18-33)$ & $23.2 \pm 3.9(19-31)$ & $0.78^{\mathrm{a}}$ \\
\hline DM & 15 & 2 & $0.89^{b}$ \\
\hline \multicolumn{4}{|l|}{ Adverse events with pregabalin } \\
\hline Somnolence & $92(56.4 \%)$ & $5(20.8 \%)$ & $0.0017^{b}$ \\
\hline Dizziness & $39(23.9 \%)$ & $11(45.8 \%)$ & $0.015^{b}$ \\
\hline Edema & $4(2.5 \%)$ & $3(12.5 \%)$ & $0.012^{b}$ \\
\hline Weight gain & $3(1.8 \%)$ & $0(0 \%)$ & $0.51^{b}$ \\
\hline Others & $2(1.2 \%)$ & $2(8.3 \%)$ & \\
\hline Lack of efficacy with pregabalin & $27(16.6 \%)$ & $5(20.8 \%)$ & $0.61^{b}$ \\
\hline NeP score (points) & $7.0 \pm 1.7(6-12)$ & $6.9 \pm 1.7(6-12)$ & $0.80^{b}$ \\
\hline Primary dose of mirogabalin (mg) & $3.1 \pm 1.2(5-10)$ & $3.3 \pm 1.8(5-10)$ & $0.52^{b}$ \\
\hline \multicolumn{4}{|l|}{ Adverse events with mirogabalin } \\
\hline Somnolence & $44(27 \%)$ & $6(25 \%)$ & $0.84^{b}$ \\
\hline Dizziness & $16(9.8 \%)$ & $7(29.2 \%)$ & $0.0069^{b}$ \\
\hline Edema & $7(4.3 \%)$ & $4(16.7 \%)$ & $0.016^{b}$ \\
\hline Epigastric pain & $0(0 \%)$ & $2(8.3 \%)$ & $<0.0001^{\mathrm{b}}$ \\
\hline Weight gain & $1(0.6 \%)$ & $0(0 \%)$ & $0.70^{b}$ \\
\hline Fatigue & $1(0.6 \%)$ & $0(0 \%)$ & $0.70^{\mathrm{b}}$ \\
\hline
\end{tabular}

Data are expressed as the mean \pm standard deviation (range) or $n(\%)$.

LCS lumbar canal stenosis, CSM cervical spondylotic myelopathy, $L D H$ lumbar disc herniation, CTS carpal tunnel syndrome, $B M I$ body mass index, DM diabetes mellitus, NeP neuropathic pain

a Student's t-test

${ }^{\mathrm{b}}$ Chi-squared test 


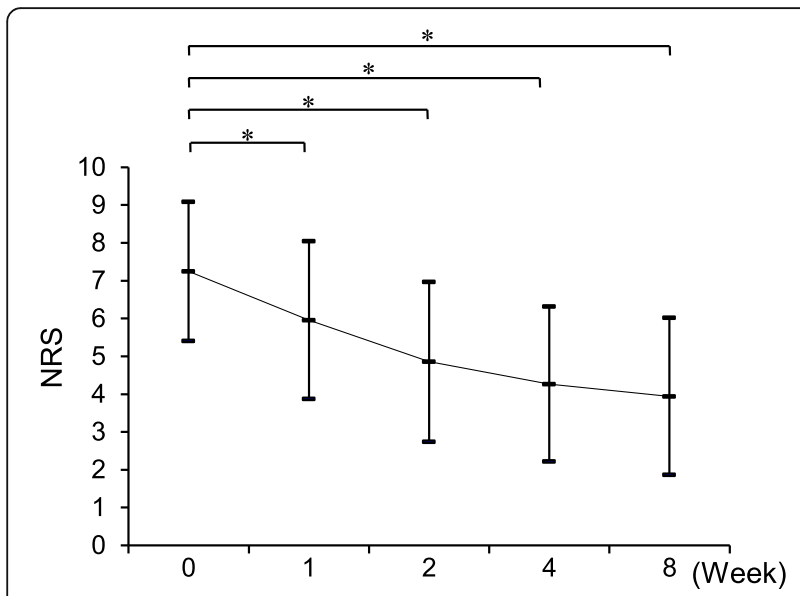

Fig. 1 Scores on the numeric rating scale (NRS) were significantly reduced after 8 weeks of treatment with mirogabalin. Data are expressed as the mean \pm standard deviation. ${ }^{*} p<0.05$

who experienced greater levels of pain relief $(p=0.0047)$. We examined whether there were differences in the adverse events and magnitude of NRS improvement between the patients with initial doses of $5 \mathrm{mg} /$ day $(n=$ $128)$ and $10 \mathrm{mg} /$ day $(n=59)$. The incidence of adverse

Table 4. Univariate analyses of patients with or without 30\% pain relief by mirogabalin

\begin{tabular}{|c|c|c|c|}
\hline Variables & $\begin{array}{l}<30 \% \text { pain relief } \\
(n=50)\end{array}$ & $\begin{array}{l}\geq 30 \% \text { pain relief } \\
(n=113)\end{array}$ & $p$ value \\
\hline Age (years) & $72.9 \pm 11.9(45-88)$ & $71.3 \pm 13.0(30-94)$ & $0.46^{\mathrm{a}}$ \\
\hline Sex, female/male & $30 / 20$ & $82 / 31$ & $0.11^{\mathrm{b}}$ \\
\hline Diagnosis & & & $0.75^{\mathrm{b}}$ \\
\hline LCS & 35 & 83 & \\
\hline CSM & 11 & 17 & \\
\hline $\mathrm{LDH}$ & 2 & 7 & \\
\hline CTS & 2 & 6 & \\
\hline Others & 0 & 1 & \\
\hline BMI $\left(\mathrm{kg} / \mathrm{m}^{2}\right)$ & $22.7 \pm 4.2(18-33)$ & $23.0 \pm 3.8(19-31)$ & $0.79^{a}$ \\
\hline DM & 4 & 11 & $0.72^{b}$ \\
\hline NeP score (points) & $7.4 \pm 2.1(6-12)$ & $6.6 \pm 1.4(6-12)$ & $0.0047^{\mathrm{a}}$ \\
\hline Primary doze (mg) & $3.1 \pm 1.5(5-10)$ & $3.1 \pm 1.1(5-10)$ & $0.97^{\mathrm{a}}$ \\
\hline Max dose (mg) & $6.6 \pm 3.8(5-30)$ & $6.4 \pm 3.8(5-30)$ & $0.76^{a}$ \\
\hline Adverse events & $24(48 \%)$ & $42(37.2 \%)$ & $0.13^{b}$ \\
\hline Somnolence & 17 (34\%) & $27(23.9 \%)$ & \\
\hline Dizziness & $6(12 \%)$ & $10(8.8 \%)$ & \\
\hline Edema & $2(4 \%)$ & $5(4.4 \%)$ & \\
\hline Weight gain & $1(2 \%)$ & $0(0 \%)$ & \\
\hline
\end{tabular}

Data are expressed as the mean \pm standard deviation (range) or $n(\%)$. LCS lumbar canal stenosis, CSM cervical spondylotic myelopathy, $L D H$ lumbar disc herniation, CTS carpal tunnel syndrome, BMI body mass index, DM diabetes mellitus, $\mathrm{NeP}$ neuropathic pain

a Student's t-test

${ }^{\mathrm{b}}$ Chi-squared test events in patients with an initial dose of $5 \mathrm{mg} /$ day (50\%, $64 / 128$ patients) was not significantly different from that in patients with $10 \mathrm{mg} /$ day $(45.8 \%, 27 / 59$ patients; $p=$ 0.59 ). There were no significant differences in the magnitude of NRS improvement between the groups with initial doses of $5 \mathrm{mg} /$ day $(46.5 \% \pm 24.8 \%)$ and $10 \mathrm{mg} /$ day $(45.5 \% \pm 24.4 \% ; p=0.81)$. Similarly, there were no significant differences in the magnitude of NRS improvement in patients taking maximum doses of $5 \mathrm{mg} /$ day $(n=47)$, $10 \mathrm{mg} /$ day $(n=89), 20 \mathrm{mg} /$ day $(n=32)$, and $30 \mathrm{mg} /$ day $(n=19, p=0.79$; Fig. 2).

\section{Discussion}

In this study, we used mirogabalin to treat patients with peripheral neuropathic pain who switched from pregabalin treatment to mirogabalin treatment due to lack of efficacy or adverse events. Mirogabalin is a new drug for the treatment of peripheral neuropathic pain, and to our knowledge, there have been no reports of its clinical outcomes for patients who were previously treated with pregabalin. Mirogabalin exerted a significant analgesic effect within 1 week and was associated with mild CNS adverse effects.

Guidelines for the pharmacologic management of neuropathic pain recommend drugs acting at $\alpha 2 \delta$ subunits of voltage-gated calcium channels, serotonin-norepinephrine reuptake inhibitors, and tricyclic antidepressants as first-line agents, neurotropin and tramadol as second-line agents, and opioids as third-line agents [25]. Several other

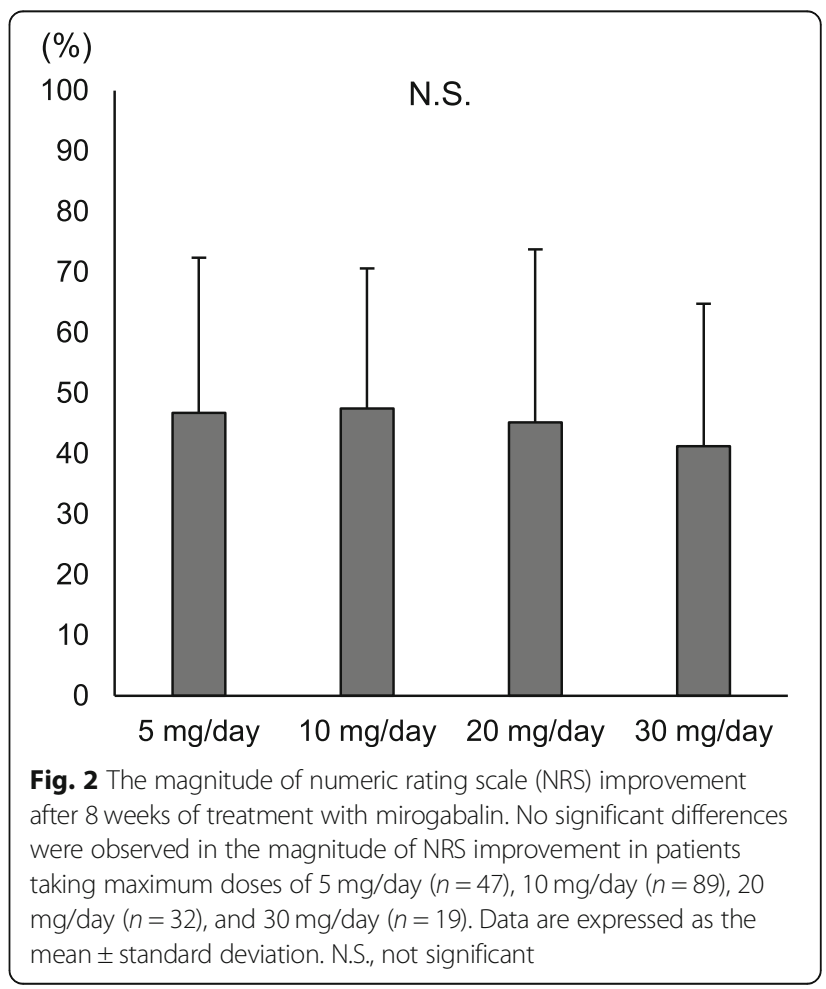


scientific associations and guidelines recommend gabapentinoids as first-line drugs for the treatment of neuropathic pain [26-29]. Mirogabalin, a ligand for the $\alpha 2 \delta$ subunits $(\alpha 2 \delta-1$ and $\alpha 2 \delta-2)$ of voltage-sensitive calcium channels in the CNS, was approved as a medication for pain relief in patients with peripheral neuropathic pain in 2019 in Japan. Mirogabalin reportedly relieved diabetic peripheral neuropathic pain in a dose-dependent manner in Asian patients with diabetic peripheral neuropathic pain and was associated with only mild adverse events [30]. It was also shown to be effective and well tolerated in the management of postherpetic neuralgia in Asian patients [31]. In the present study, which included only patients who withdrew from pregabalin treatment due to adverse events or lack of efficacy, we found that mirogabalin was an effective analgesic and that few patients stopped treatment due to adverse events.

In vitro studies of its pharmacologic action have demonstrated that mirogabalin had a higher binding affinity for the human and rat $\alpha 2 \delta$ subunit than pregabalin [16]. In a dissociation rate analysis, the dissociation half-lives of mirogabalin from the $\alpha 2 \delta-1$ and $\alpha 2 \delta-2$ subunits were $11.1 \mathrm{~h}$ and $2.4 \mathrm{~h}$, respectively, compared with $1.4 \mathrm{~h}$ for pregabalin at both subunits [16]. These reports indicated that mirogabalin has potent and selective binding affinities for the human and rat $\alpha 2 \delta$ subunit and a slower dissociation rate for the $\alpha 2 \delta-1$ subunit than the $\alpha 2 \delta-2$ subunit compared with pregabalin [16]. These findings support our results that even patients who discontinued pregabalin because of its CNS effects experienced fewer adverse events when they were treated with mirogabalin. In this study, the initial dose was reduced to $5 \mathrm{mg} /$ day in patients with impaired renal function or in the elderly patients. In patients with impaired renal function, we consider that the initial dose of $10 \mathrm{mg} /$ day was too high, which may cause adverse events. However, in this study, the incidence of adverse events was nearly the same in the patients with impaired renal function as in the patients with normal renal function, and there was no significant difference in the magnitude of NRS improvement after 8 weeks of treatment. Therefore, we consider that administering a reduced dose is useful in patients with impaired renal function. It has also been reported that continued oral mirogabalin treatment increases the pain threshold over time [16]. In animal models of fibromyalgia, mirogabalin treatment has been shown to significantly decrease pain scores due to chronic allodynia [32]. Neuropathic pain results in higher pain scores than nociceptive pain [4]. In this study, the dose of mirogabalin was increased in response to pain over an 8-week treatment period. The magnitude of NRS improvement was $40 \%$ or more at the $10 \mathrm{mg} /$ day, $20 \mathrm{mg} /$ day, and $30 \mathrm{mg} /$ day doses, and the analgesic effect of mirogabalin was evident after only 1 week, suggesting a good analgesic effect of mirogabalin.
The $\alpha 2 \delta-1$ subunit plays an important role in the onset and pathological persistence of neuropathic pain. A2 $\delta$ - 1 expression levels correlated with tactile allodynia development were significantly increased in rats with spinal cord injury [33]. Knockdown of the $\alpha 2 \delta-1$ subunit by antisense oligodeoxynucleotides reportedly inhibited tactile allodynia in rat models [33, 34]. Overexpression of the $\alpha 2 \delta-1$ subunit resulted in enhanced currents, altered kinetics, and voltage-dependence of voltage-gated calcium channel activation in sensory neurons; exaggerated and prolonged dorsal horn neuronal responses to mechanical and thermal stimulations in the periphery; and enhanced pain-related behavior [35]. To the best of our knowledge, no study has addressed the association between the $\alpha 2 \delta-2$ subunit and pain. Edvardson et al. reported the importance of the $\alpha 2 \delta-2$ subunit, which is dominantly expressed in cerebellar Purkinje cells, in the normal physiology of the human brain [36]. Binding to the $\alpha 2 \delta-1$ subunit contributes to analgesic effects, whereas binding to the $\alpha 2 \delta-2$ subunit appears to contribute to undesirable CNS effects, such as somnolence [37-39]. These studies indicated that gabapentinoids exert their analgesic effects via the $\alpha 2 \delta-1$ subunit, and the $\alpha 2 \delta-1$ subunit thus plays a major role in neuropathic pain. These findings suggested that the $\alpha 2 \delta-2$ subunit may be implicated in the CNS adverse events commonly seen with pregabalin treatment. These findings also suggested that the selective actions of mirogabalin on the $\alpha 2 \delta-1$ and $\alpha 2 \delta-2$ subunits may maximize its analgesic effects while minimizing CNS adverse events. The potent binding affinity of mirogabalin with the $\alpha 2 \delta-1$ subunit and its long dissociation half-life from the $\alpha 2 \delta-2$ subunit may thus make mirogabalin an attractive agent for the treatment of peripheral neuropathic pain. Although $12.8 \%$ of patients in the present study discontinued treatment because of adverse events, mirogabalin was generally well tolerated.

Although the results of the present study suggest that mirogabalin might be an alternative treatment option for the treatment of peripheral neuropathic pain, the present study has some limitations. First, both pregabalin and mirogabalin are ligands for the $\alpha 2 \delta$ subunit of voltagesensitive calcium channels. However, this study had no pretrial protocol and is a case series without a comparison or placebo group. We did not compare these two drugs in this study. Thus, the results cannot be clearly attributed to mirogabalin administration. Second, this study had a short observation period. Although most patients treated with mirogabalin for 8 weeks maintained their weight within $\pm 5 \%$ of their baseline weight, weight gain can be an issue with mirogabalin when used for a longer period. A third limitation was a lack of determination of the best screening questionnaire for neuropathic pain. Neuropathic pain screening questionnaires include 
painDETECT [4], the spine painDETECT questionnaire, which is a screening tool for neuropathic pain caused by spinal disorders [40], and the $\mathrm{NeP}$ score used in this study. We considered that if the $\mathrm{NeP}$ scores were high, there would be little decrease in NRS scores after mirogabalin treatment, making it suitable as a baseline index. However, the use of other neuropathic pain screening questionnaires might have led to different results. We consider that the assessment and diagnosis of neuropathic pain should follow an identical algorithm that is widely used as a current international standard for the diagnosis of neuropathic pain, and it should specifically include (1) an assessment of the range of pain that is neuroanatomically plausible, (2) the suggestion of a lesion or disease of the somatosensory system, and (3) objective findings of sensory damage that are observed in the neuroanatomically innervated region of the damaged nerve or tests that are performed to provide a diagnosis of a neurological lesion or disease that accounts for the neuropathic pain. Despite these limitations, mirogabalin, a recently developed agent, showed promising results in patients with peripheral neuropathic pain.

\section{Conclusions}

This investigation indicated that mirogabalin is safe and effective for reducing peripheral neuropathic pain in patients who ceased treatment with pregabalin due to adverse events or lack of efficacy.

\section{Abbreviations}

QOL: Quality of life; CNS: Central nervous system; NRS: Numeric rating scale; BMl: Body mass index

\section{Acknowledgements}

We would like to thank American Journal Experts for English language editing.

\section{Authors' contributions \\ TomokT, TomonT, KN, and TO did the conceptualization. TomokT did the data curation. TomokT and TomonT did formal analysis. TomokT did the investigation. TomokT, TomonT, HM, TTa, KY, HT, and TO did the methodology. TomonT, HM, TTa, and KY did supervision. TomokT and TomonT wrote the original draft. TomokT, TomonT, KN, HM, TTa, KY, HT, and TO. did the review and editing. The authors read and approved the final manuscript.}

\section{Funding}

This research was supported by Grants-in-Aid for Scientific Research (17K16691).

\section{Availability of data and materials}

All data used and analyzed during this study are available from the corresponding author upon reasonable request.

\section{Ethics approval and consent to participate}

The Kurashiki Municipal Hospital ethics committee approved this research.

\section{Consent for publication}

Not applicable

\section{Competing interests}

The authors declare that they have no competing interests.

\section{Author details}

'Department of Orthopedic Surgery, Okayama University Hospital, Okayama, Japan, 2-5-1 Shikata-cho, Kitaku, Okayama City, Okayama 700-8558, Japan. ${ }^{2}$ Department of Orthopedic Surgery, Kurashiki Municipal Hospital, 2-39, Kojima-Ekimae, Kurashiki 711-0921, Japan.

Received: 1 February 2020 Accepted: 13 May 2020

Published online: 26 May 2020

\section{References}

1. Jensen TS, Baron R, Haanpaa M, Kalso E, Loeser JD, Rice AS, et al. A new definition of neuropathic pain. Pain. 2011;152:2204-5. https://doi.org/10. 1016/j.pain.2011.06.017.

2. O'Connor AB. Neuropathic pain: quality-of-life impact, costs and cost effectiveness of therapy. Pharmacoeconomics. 2009;27:95-112. https://doi. org/10.2165/00019053-200927020-00002.

3. Baron R, Binder A. [How neuropathic is sciatica? The mixed pain concept]. Orthopade. 2004;33:568-75. doi: 10.1007/s00132-004-0645-0 [doi].

4. Freynhagen R, Baron R. Gockel U. Tolle TR. painDETECT: a new screening questionnaire to identify neuropathic components in patients with back pain. Curr Med Res Opin. 2006;22:1911-20. https://doi.org/10.1185/ $030079906 \times 132488$.

5. Alles SRA, Smith PA. Etiology and pharmacology of neuropathic pain. Pharmacol Rev. 2018;70:315-47. https://doi.org/10.1124/pr.117.014399.

6. Cruccu G, Truini A. A review of neuropathic pain: from guidelines to clinical practice. Pain Ther. 2017;6:35-42. https://doi.org/10.1007/s40122-017-0087-0,

7. Kumar N, Laferriere A, Yu JS, Leavitt A, Coderre TJ. Evidence that pregabalin reduces neuropathic pain by inhibiting the spinal release of glutamate. J Neurochem. 2010;113:552-61. https://doi.org/10.1111/j.1471-4159.2010. 06625.x.

8. Akazawa M, Igarashi A, Ebata N, Murata T, Zeniya S, Haga Y, et al. A costeffectiveness analysis of pregabalin for the treatment of patients with chronic cervical pain with a neuropathic component in Japan. J Pain Res. 2019;12:2785-97. https://doi.org/10.2147/jpr.S203712.

9. Igarashi A, Akazawa M, Murata T, Taguchi T, Sadosky A, Ebata N, et al. Costeffectiveness analysis of pregabalin for treatment of chronic low back pain in patients with accompanying lower limb pain (neuropathic component) in Japan. Clinicoecon Outcomes Res. 2015;7:505-20. https://doi.org/10.2147/ ceor.S89833.

10. Gianesello L, Pavoni V, Barboni E, Galeotti I, Nella A. Perioperative pregabalin for postoperative pain control and quality of life after major spinal surgery. $J$ Neurosurg Anesthesiol. 2011. doi: 10.1097/ANA.0b013e31823a885b [doi].

11. Onakpoya IJ, Thomas ET, Lee JJ, Goldacre B, Heneghan CJ. Benefits and harms of pregabalin in the management of neuropathic pain: a rapid review and meta-analysis of randomised clinical trials. BMJ Open. 2019;9: e023600. https://doi.org/10.1136/bmjopen-2018-023600.

12. Hindmarch I, Trick L, Ridout F. A double-blind, placebo- and positive-internalcontrolled (alprazolam) investigation of the cognitive and psychomotor profile of pregabalin in healthy volunteers. Psychopharmacology (Berl). 2005;183:13343. doi: 10.1007/s00213-005-0172-7 [doi].

13. Rosenstock J, Tuchman M, LaMoreaux L, Sharma U. Pregabalin for the treatment of painful diabetic peripheral neuropathy: a double-blind placebo-controlled trial. Pain. 2004;110:628-38. doi: 10.1016/j.pain.2004.05. 001 [doi] S0304395904002465 [pii].

14. Baidya DK, Agarwal A, Khanna P, Arora MK. Pregabalin in acute and chronic pain. J Anaesthesiol Clin Pharmacol. 2011;27:307-14. https://doi.org/10.4103/ 0970-9185.83672.

15. Deeks ED. Mirogabalin: first global approval. Drugs. 2019;79:463-8. https:// doi.org/10.1007/s40265-019-01070-8.

16. Domon $\mathrm{Y}$, Arakawa N, Inoue T, Matsuda F, Takahashi M, Yamamura N, et al. Binding characteristics and analgesic effects of mirogabalin, a novel ligand for the a $2 \delta$ subunit of voltage-gated calcium channels. J Pharmacol Exp Ther. 2018;365:573-82. https://doi.org/10.1124/jpet.117.247551.

17. Tetsunaga T, Tetsunaga T, Tanaka M, Sugimoto Y, Takigawa T, Ozaki T. Outcomes of pregabalin in lumbar-disease patients with depression. J Orthop Sci. 2014;19:229-34. https://doi.org/10.1007/s00776-013-0529-4.

18. Treede RD, Jensen TS, Campbell JN, Cruccu G, Dostrovsky JO, Griffin JW, et al. Neuropathic pain: redefinition and a grading system for clinical and research purposes. Neurology. 2008;70:1630-5. https://doi.org/10.1212/01. wnl.0000282763.29778.59. 
19. Sakai Y, Ito K, Hida T, Ito S, Harada A. Neuropathic pain in elderly patients with chronic low back pain and effects of pregabalin: a preliminary study. Asian Spine J. 2015;9:254-62. https://doi.org/10.4184/asj.2015.9.2.254.

20. Yamashita T, Takahashi K, Yonenobu K, Kikuchi S. Prevalence of neuropathic pain in cases with chronic pain related to spinal disorders. J Orthop Sci. 2014;19:15-21. https://doi.org/10.1007/s00776-013-0496-9.

21. Ogawa S. Development of new screening questionnaire to identify neuropathic components in Japanese patients with chronic pain. Pain Clinic. 2010;31:1187-94

22. Jensen MP, Karoly P, Braver S. The measurement of clinical pain intensity: a comparison of six methods. Pain. 1986;27:117-26 doi: 0304-3959(86)90228-9 [pii].

23. Javed S, Petropoulos IN, Alam U, Malik RA. Treatment of painful diabetic neuropathy. Ther Adv Chronic Dis. 2015;6:15-28. https://doi.org/10.1177/ 2040622314552071.

24. Javed S, Alam U, Malik RA. Mirogabalin and emerging therapies for diabetic neuropathy. J Pain Res. 2018;11:1559-66. https://doi.org/10.2147/jpr.S145999.

25. The Committee for the Guidelines for the Pharmacologic Management of Neuropathic Pain of JSPC. Guidelines for the Pharmacologic Management of Neuropathic Pain Second Edition: Publication Department, Shinko Trading Co. Ltd.; 2016.

26. Dworkin RH, O'Connor AB, Kent J, Mackey SC, Raja SN, Stacey BR, et al. Interventional management of neuropathic pain: NeuPSIG recommendations. Pain. 2013;154:2249-61. https://doi.org/10.1016/j.pain 2013.06.004

27. Bril V, England J, Franklin GM, Backonja M, Cohen J, Del Toro D, et al. Evidence-based guideline: treatment of painful diabetic neuropathy: report of the American Academy of Neurology, the American Association of Neuromuscular and Electrodiagnostic Medicine, and the American Academy of Physical Medicine and Rehabilitation. Neurology. 2011;76:1758-65. https://doi.org/10.1212/WNL.0b013e3182166ebe.

28. Attal N, Cruccu G, Baron R, Haanpaa M, Hansson P, Jensen TS, et al. EFNS guidelines on the pharmacological treatment of neuropathic pain: 2010 revision. Eur J Neurol. 2010;17:1113-e88. https://doi.org/10.1111/j.1468-1331. 2010.02999.x.

29. Moulin D, Boulanger A, Clark AJ, Clarke H, Dao T, Finley GA, et al. Pharmacological management of chronic neuropathic pain: revised consensus statement from the Canadian Pain Society. Pain Res Manag 2014;19:328-35. https://doi.org/10.1155/2014/754693.

30. Baba M, Matsui N, Kuroha M, Wasaki Y, Ohwada S. Mirogabalin for the treatment of diabetic peripheral neuropathic pain: a randomized, doubleblind, placebo-controlled phase III study in Asian patients. J Diabetes Investig. 2019;10:1299-306. https://doi.org/10.1111/jdi.13013.

31. Kato J, Matsui N, Kakehi Y, Murayama E, Ohwada S, Sugihara M. Mirogabalin for the management of postherpetic neuralgia: a randomized, double-blind, placebo-controlled phase 3 study in Asian patients. Pain. 2019;160:1175-85. https://doi.org/10.1097/j.pain.0000000000001501.

32. Saeki K, Yasuda SI, Kato M, Kano M, Domon Y, Arakawa N, et al. Analgesic effects of mirogabalin, a novel ligand for a $2 \delta$ subunit of voltage-gated calcium channels, in experimental animal models of fibromyalgia. Naunyn Schmiedebergs Arch Pharmacol. 2019;392:723-8. https://doi.org/10.1007/ s00210-019-01628-z.

33. Boroujerdi A, Zeng J, Sharp K, Kim D, Steward O, Luo ZD. Calcium channel alpha-2-delta-1 protein upregulation in dorsal spinal cord mediates spinal cord injury-induced neuropathic pain states. Pain. 2011;152:649-55. https:// doi.org/10.1016/j.pain.2010.12.014.

34. Li CY, Song YH, Higuera ES, Luo ZD. Spinal dorsal horn calcium channel a2 $\delta$-1 subunit upregulation contributes to peripheral nerve injury-induced tactile allodynia. J Neurosci. 2004;24:8494-9. https://doi.org/10.1523/ jneurosci.2982-04.2004

35. Li CY, Zhang XL, Matthews EA, Li KW, Kurwa A, Boroujerdi A, et al. Calcium channel a $2 \delta 1$ subunit mediates spinal hyperexcitability in pain modulation. Pain. 2006;125:20-34. https://doi.org/10.1016/j.pain.2006.04.022.

36. Edvardson S, Oz S, Abulhijaa FA, Taher FB, Shaag A, Zenvirt S, et al. Early infantile epileptic encephalopathy associated with a high voltage gated calcium channelopathy. J Med Genet. 2013;50:118-23. https://doi.org/10. 1136/jmedgenet-2012-101223.

37. Field MJ, Cox PJ, Stott E, Melrose H, Offord J, Su TZ, et al. Identification of the a 2- $\delta-1$ subunit of voltage-dependent calcium channels as a molecular target for pain mediating the analgesic actions of pregabalin. Proc Natl Acad Sci U S A. 2006:103:17537-42. https://doi.org/10.1073/pnas. 0409066103.
38. Barclay J, Balaguero N, Mione M, Ackerman SL, Letts VA, Brodbeck J, et al. Ducky mouse phenotype of epilepsy and ataxia is associated with mutations in the Cacna2d 2 gene and decreased calcium channel current in cerebellar Purkinje cells. J Neurosci. 2001;21:6095-104.

39. Vinik A, Rosenstock J, Sharma U, Feins K, Hsu C, Merante D. Efficacy and safety of mirogabalin (DS-5565) for the treatment of diabetic peripheral neuropathic pain: a randomized, double-blind, placebo- and active comparator-controlled, adaptive proof-of-concept phase 2 study. Diabetes Care. 2014;37:3253-61. https://doi.org/10.2337/dc14-1044

40. Nikaido T, Sumitani M, Sekiguchi M, Konno S. The Spine painDETECT questionnaire: development and validation of a screening tool for neuropathic pain caused by spinal disorders. PLoS One. 2018;13:e0193987. https://doi.org/10.1371/journal.pone.0193987.

\section{Publisher's Note}

Springer Nature remains neutral with regard to jurisdictional claims in published maps and institutional affiliations.
Ready to submit your research? Choose BMC and benefit from:

- fast, convenient online submission

- thorough peer review by experienced researchers in your field

- rapid publication on acceptance

- support for research data, including large and complex data types

- gold Open Access which fosters wider collaboration and increased citations

- maximum visibility for your research: over $100 \mathrm{M}$ website views per year

At $\mathrm{BMC}$, research is always in progress.

Learn more biomedcentral.com/submissions 